\title{
PROPAGATION OF EXPLOSIVE SOUND IN A LIQUID LAYER OVERLYING A SEMI-INFINITE ELASTIC SOLID*
}

\author{
FRANK PRESS $†$ AND MAURICE EWING $\dagger$
}

\section{ABSTRACT}

The Pekeris theory of normal mode propagation of explosion sound in two liquid layers is extended to include the case of a solid bottom. Curves giving phase velocity, group velocity, amplitude, vertical pressure distribution as a function of frequency are presented. The relative merits of geophones and hydrophones for underwater seismology and the best depth for each type of instrument are discussed in the light of the theory. The characteristics of an incoming signal are described.

\section{INTRODUCTION}

The normal mode theory of sound propagation in two and three liquid layers was given by Pekeris (1948) who solved the problem for waves originating at an impulsive point source located in the first layer. Pekeris showed conclusively that his solution fully explained most of the characteristics of explosive sounds in shallow water observed by Worzel and Ewing (1948).

The plane wave solution for sound propagation in three liquid layers in which the speed of sound in the intermediate layer is less than that in the topmost layer was investigated by Press and Ewing (1948a).

The treatment of the ocean bottom as a liquid was adequate for the shallow water data to which Pekeris applied it since the penetration into the bottom is approximately equal to the depth of water which usually involves only unconsolidated sediments. When one deals with sound propagation in the deep ocean or in shallow water underlain by rock bottom, it becomes necessary to consider the bottom as an elastic solid. Several of our recent papers have dealt with certain aspects of propagation in a liquid layer overlying an elastic solid, and in the present paper the Pekeris theory is extended to include the case of normal mode sound propagation from an impulsive point source in a liquid layer overlying a semi-infinite elastic solid. Certain derivations covered in the liquid bottom theory will not be repeated here, but reference will be made to the paper of Pekeris.

Early work by Stoneley (1926) and Sezawa (1934) dealt with the effect of the ocean on the transmission of seismic waves across a solid ocean bottom. Stoneley considered plane waves and calculated several values of phase and group velocity for long periods. Sezawa obtained an approximate solution for the propagation of cylindrical waves having great length compared to the depth of water. Lee (r934) calculated the amplitudes of Rayleigh waves for plane waves transmitted

* Presented at the St. Louis Meeting of the Society March 1949. Contribution No. II, Lamont Geological Observatory (Columbia University) Palisades, New York. Manuscript received by the Editor February 6, 1950 .

† Lamont Geological Observatory, Columbia University, Palisades, New York. 
through an elastic solid covered by a superficial surface layer, assuming several values for the elastic constants of the two media.

Scholte (I 943) gave a theory for the combined effects of gravity and compressibility in a layer of water in contact with an elastic solid bottom. Scholte's emphasis was on energy transfer from the atmosphere to the ocean bottom and he did not deal with group velocity or pay particular attention to transmission in a horizontal direction. Press and Ewing (1948b) presented curves of phase and group velocity for the first and second modes of motion of plane waves in a liquid layer superposed on a solid bottom and later (Press, et al.) extended the theoretical work of Lamb (I904) and Pekeris (1948) to include the case of an impulsive point source of compressional waves located within the solid bottom.

THEORY

\section{Sleady Slate Solution}

Consider the propagation of elastic waves through the system consisting of a liquid layer of thickness $H$, density $\rho_{1}$ and compressional wave velocity $v_{1}$, superposed upon an infinitely thick, solid bottom of density $\rho_{2}$, compressional wave velocity $\alpha_{2}$ and distortional wave velocity $\beta_{2}$. The incompressibility of the liquid is $\lambda_{1}$, and $\lambda_{2}, \mu_{2}$ are Lame's constants for the bottom, and are related to the elastic wave velocities as follows:

$$
\left.\begin{array}{rl}
v_{1}{ }^{2} & =\lambda_{1} / \rho_{1} \\
\alpha_{2}{ }^{2} & =\left(\lambda_{2}+2 \mu_{2}\right) / \rho_{2} \\
\beta_{2}{ }^{2} & =\mu_{2} / \rho_{2}
\end{array}\right\} .
$$

The cartesian coordinate system is chosen with the $x$ and $y$ axes in the liquid surface and the $z$ axis vertically downward. We will use the coordinates $z$ and $r=\sqrt{x^{2}+y^{2}}$ and denote the corresponding displacements by $q$ and $w$ respectively. The subscripts $\mathrm{I}$ and 2 refer to the liquid layer and solid bottom respectively.

\section{Simple Harmonic Point Source within Liquid Layer}

We wish to determine the velocity potential $\phi_{1}$ from which the component displacements $q_{1}$ and $w_{1}$ and the pressure $p_{1}$ due to a point source of compressional waves at $r=0, z=d$ can be obtained. In particular we will seek the normal mode solutions which predominate at large distances from the source.

We follow the procedure of Lamb (1904) in assuming simple harmonic motion $e^{i w t}$ and introducing the functions $\phi(r, z)$ and $\psi(r, z)$ defined by the equations:

$$
\left.\begin{array}{rl}
q_{1} & =\partial \phi_{1} / \partial r \\
w_{1} & =\partial \phi_{1} / \partial z \\
q_{2} & =\partial \phi_{2} / \partial r+\partial^{2} \psi_{2} / \partial r \partial z \\
\mathfrak{w}_{2} & =\partial \phi_{2} / \partial z+\partial^{2} \psi_{2} / \partial z^{2}+\omega^{2} / \beta_{2}{ }^{2} \psi_{2}
\end{array}\right\}
$$


The vertical stress $p_{z z}$ and the tangential stress $p_{z r}$ can be expressed in terms of $q, w, \phi, \psi$ and the elastic constants as follows:

$$
\begin{aligned}
& p_{z z}=\lambda \nabla^{2} \phi+2 \mu \partial w / \partial z \\
& p_{z r}=\mu(\partial q / \partial z+\partial w / \partial r) .
\end{aligned}
$$

It is required that the functions $\phi$ and $\psi$ satisfy the wave equations (reduced for simple harmonic motion):

$$
\begin{aligned}
& \left(\nabla^{2}+\omega^{2} / v_{1}^{2}\right) \phi_{1}=0 \\
& \left(\nabla^{2}+\omega^{2} / \alpha_{2}^{2}\right) \phi_{2}=0 \\
& \left(\nabla^{2}+\omega^{2} / \beta_{2}^{2}\right) \psi_{2}=0
\end{aligned}
$$

where

$$
\nabla^{2}=\dot{\partial}^{2} / \partial r^{2}+\frac{1}{r} \partial / \partial r+\partial^{2} / \partial z^{2}
$$

Solutions of (5), (6), and (7) must satisfy the boundary conditions:

$$
\begin{aligned}
& \left(p_{z z}\right)_{1}=0 \quad \text { at } \quad z=0 \\
& \left(p_{z z}\right)_{1}^{\prime}=\left(p_{z z}\right)_{2} \text { at } z=H \\
& \left(p_{2 r}\right)_{2}=0 \text { at } z=H \\
& w_{1}^{\prime}=w_{2} \text { at } z=H \text {. }
\end{aligned}
$$

The meaning of the primed symbols is given in the next paragraph.

Our procedure will be to first obtain the solutions to the problem where a periodic pressure is applied to the entire plane $z=d$ symmetrically about the $z$ axis and then to pass to the case of a point source utilizing the Fourier-Bessel integral. We shall represent a point source located at $r=0, z=d$ by requiring continuity of pressure in the plane $z=d$ and continuity of vertical displacement $w$ everywhere in the same plane except at the point source where the fluid above and below the source moves in opposite directions, the discontinuity in $w$ here becoming proportional to a function $F(r)$ which vanishes everywhere except at $r=0$ where it becomes infinite in such a manner that its integral over the plane $z=d$ is finite. It is convenient to divide the liquid layer into regions above and below this plane indicated by primed and unprimed symbols respectively.

Typical solutions of $(5),(6)$, and $(7)$ are of the form:

$$
\begin{aligned}
\phi_{1} & =A \sin (\xi z) J_{0}(k r) \\
\phi_{1}^{\prime} & =[B \sin (\xi z)+C \cos (\xi z)] J_{0}(k r) \\
\phi_{2} & =D e^{-\eta z} J_{0}(k r) \\
\psi_{2} & =E e^{-\zeta z} J_{0}(k r)
\end{aligned}
$$


where the separation constants $\xi, \eta, \zeta$, obtained by substituting (I2)-(15) in $(5)-(7)$ are

$$
\xi^{2}=\omega^{2} / v_{1}{ }^{2}-k^{2}, \quad \eta^{2}=k^{2}-\omega^{2} / \alpha_{2}{ }^{2}, \quad \zeta^{2}=k^{2}-\omega^{2} / \beta_{2}{ }^{2}
$$

$\xi, \eta, \zeta$ are positive real or negative imaginary following the convention adopted by Pekeris. (I 948 ).

These solutions must satisfy two additional boundary conditions at the plane $z=d$. We require continuity of pressure

$$
\left(p_{z z}\right)_{1}=\left(p_{z z}\right)_{1}^{\prime} \text { at } z=d
$$

and a discontinuity in vertical displacement given by

$$
\left(\partial \phi_{1} / \partial z\right)-\left(\partial \phi_{1} / \partial z\right)^{\prime}={ }_{2} Z J_{0}(k r)
$$

the fluid above and below the plane moving in opposite directions.

The expression for $\phi_{1}{ }^{\prime}$ is the general form. $\phi_{1}$ has been chosen to satisfy the first boundary condition (8). The functions $\phi_{2}$ and $\psi_{2}$ are taken to decrease exponentially with depth since we are interested in solutions for which the loss of energy by refraction into the bottom is zero.

The constants $A, B, C, D, E$ determined by substituting equations (I2)(I5) in the boundary condition equations (9)-(II) and (I7)-(I8) are as follows:

$$
\begin{aligned}
& A=\frac{2 Z}{\xi} \frac{\left\{\frac{\rho_{1}}{\rho_{2}} \frac{\omega^{4}}{\beta_{2}{ }^{4}} \frac{\eta}{\xi} \sin \xi(H-d)-\left[4 k^{2} \eta \zeta-\left(2 k^{2}-\omega^{2} / \beta_{2}{ }^{2}\right)^{2}\right] \cos \xi(H-d)\right\}}{\left\{\frac{\rho_{1}}{\rho_{2}} \frac{\omega^{4}}{\beta_{2}{ }^{4}} \frac{\eta}{\xi} \sin (\xi H)-\left[4 k^{2} \eta \zeta-\left(2 k^{2}-\omega^{2} / \beta_{2}{ }^{2}\right)^{2}\right] \cos (\xi H)\right\}} \\
& B=-\frac{2 Z}{\xi} \sin (\xi d) \frac{\left\{\frac{\rho_{1}}{\rho_{2}} \frac{\omega^{4}}{\beta_{2}{ }^{4}} \frac{\eta}{\xi} \cos (\xi H)+\left[4 k^{2} \eta \zeta-\left(2 k^{2}-\omega^{2} / \beta_{2}{ }^{2}\right)^{2}\right] \sin (\xi H)\right\}}{\left\{\frac{\rho_{1}}{\rho_{2}} \frac{\omega^{2}}{\beta_{2}{ }^{4}} \frac{\eta}{\xi} \sin (\xi H)-\left[4 k^{2} \eta \zeta-\left(2 k^{2}-\omega^{2} / \beta_{2}{ }^{2}\right)^{2}\right] \cos (\xi H)\right\}}(20) \\
& C=\frac{2 Z}{\xi} \sin (\xi d) \\
& D=-\frac{{ }_{2} Z}{\xi} \sin (\xi d) \frac{\frac{\rho_{1}}{\rho_{2}} \frac{\omega^{2}}{\beta_{2}{ }^{2}}\left(2 k^{2}-\omega^{2} / \beta_{2}{ }^{2}\right) e^{\eta H}}{\left\{\frac{\rho_{1}}{\rho_{2}} \frac{\omega^{4}}{\beta_{2}{ }^{4}} \frac{\eta}{\xi} \sin (\xi H)-\left[4 k^{2} \eta \zeta-\left(2 k^{2}-\omega^{2} / \beta_{2}{ }^{2}\right)^{2}\right] \cos (\xi H)\right\}} \\
& E=-\frac{2 Z}{\xi} \sin (\xi d) \frac{2 \frac{\rho_{1}}{\rho_{2}} \frac{\omega^{2}}{\beta_{2}{ }^{2}} \eta e^{\zeta H}}{\left\{\frac{\rho_{1}}{\rho_{2}} \frac{\omega^{4}}{\beta_{2}{ }^{4}} \frac{\eta}{\xi} \sin (\xi H)-\left[4 k^{2} \eta \zeta-\left(2 k^{2}-\omega^{2} / \beta_{2}{ }^{2}\right)^{2}\right] \cos (\xi H)\right\}}(23)
\end{aligned}
$$


We now generalize the discontinuity in $w$ by means of the Fourier-Bessel integral:

$$
F(r)=\int_{0}^{\infty} J_{0}(k r) k d k \int_{0}^{\infty} F(\lambda) J_{0}(k \lambda) \lambda d \lambda
$$

and we choose $F(\lambda)$ to vanish for all but infinitesimal values of $\lambda$ in such a manner that its integral over the plane $z=d$ is unity, or

$$
\int_{0}^{\infty} F(\lambda) 2 \pi \lambda d \lambda=\text { finite. }
$$

Thus if we take $Z=k d k$ in the solutions (12)-(15) and (19)-(23) and integrate with respect to $k$ from $\circ$ to $\infty$ we obtain solutions which satisfy the conditions at the boundaries and meet the additional requirements of a point source, namely, continuity of pressure in the plane $z=d$ and continuity of vertical displacement everywhere in this plane except at $r=0$ where the discontinuity becomes proportional to $F(r)=\int J_{0}^{\infty}(k r) k d k$ which becomes infinite in such a way that its integral over the plane $z=d$ is finite.

The formal solutions for a periodic point source are therefore:

$$
\begin{aligned}
& \phi_{1}=2 e^{i \omega t} \int_{0}^{\infty} J_{0}(k r) k d k \frac{\sin (\xi z)}{\xi} \\
& \left\{\frac{\frac{\rho_{1}}{\rho_{2}} \frac{\omega^{4}}{\beta_{2}^{4}} \frac{\eta}{\xi} \sin \xi(H-d)-\left[4 k^{2} \eta \zeta-\left(2 k^{2}-\frac{\omega^{2}}{\beta_{2}^{2}}\right)^{2}\right] \cos \xi(H-d)}{\frac{\rho_{1}}{\rho_{2}} \frac{\omega^{4}}{\beta_{2}^{4}} \frac{\eta}{\xi} \sin (\xi H)-\left[4 k^{2} \eta \zeta-\left(2 k^{2}-\frac{\omega^{2}}{\beta_{2}^{2}}\right)^{2}\right] \cos (\xi H)}\right\} \\
& 0 \leqq z \leqq d \\
& \phi_{1}^{\prime}=2 e^{i \omega t} \int_{0}^{\infty} J_{0}(k r) k d k \frac{\sin (\xi d)}{\xi} \\
& \left\{\frac{\frac{\rho_{1}}{\rho_{2}} \frac{\omega^{4}}{\beta_{2}{ }^{4}} \frac{\eta}{\xi} \sin \xi(H-z)-\left[4 k^{2} \eta \zeta-\left(2 k^{2}-\frac{\omega^{2}}{\beta_{2}{ }^{2}}\right)^{2}\right] \cos \xi(H-z)}{\frac{\rho_{1}}{\rho_{2}} \frac{\omega^{4}}{\beta_{2}{ }^{4}} \frac{\eta}{\xi} \sin (\xi H)-\left[4 k^{2} \eta \zeta-\left(2 k^{2}-\frac{\omega_{2}{ }^{2}}{\beta_{2}{ }^{2}}\right)^{2}\right] \cos (\xi H)}\right\} \\
& \phi_{2}=-2 e^{i \omega \iota} \int_{0}^{\infty} J_{0}(k r) k d k \frac{\sin (\xi d)}{\xi} \\
& d \leqq z \leqq H \\
& \left\{\frac{\frac{\rho_{1}}{\rho_{2}} \frac{\omega^{2}}{\beta_{2}^{2}}\left(2 k^{2}-\frac{\omega^{2}}{\beta_{2}^{2}}\right) e^{-\eta(z-H)}}{\frac{\rho_{1}}{\rho_{2}} \frac{\omega^{4}}{\beta_{2}^{4}} \frac{\eta}{\xi} \sin (\xi H)-\left[4 k^{2} \eta \zeta-\left(2 k^{2}-\frac{\omega^{2}}{\beta_{2}^{2}}\right)^{2}\right] \cos (\xi H)}\right\} \\
& z \geqq H
\end{aligned}
$$




$$
\begin{aligned}
\psi_{2}=-2 e^{i \omega t} \int_{0}^{\infty} J_{0}(k r) k d k \frac{\sin (\xi d)}{\xi} \\
\left\{\begin{array}{c}
\frac{\rho_{1}}{\rho_{2}} \frac{\omega^{2}}{\beta_{2}{ }^{2}} \eta e^{-\zeta(z-H)} \\
\frac{\rho_{1}}{\rho_{2}} \frac{\omega^{4}}{\beta_{2}^{4}} \frac{\eta}{\xi} \sin (\xi H)-\left[4 k^{2} \eta \zeta-\left(2 k^{2}-\frac{\omega^{2}}{\beta_{2}{ }^{2}}\right)^{2}\right] \cos (\xi H)
\end{array}\right\} \\
z \geqq H .
\end{aligned}
$$

Integrals of this general type have been evaluated by Lamb, (1904), Nakano (I925), Sezawa (I935), and Pekeris (1948). The procedure is to transform the path of integration to the complex $k$-plane. The solutions can then be expressed as the sum of the residues of the integrands and two integrals along branch lines corresponding to the branch points $k=\omega / \alpha_{2}$ and $k=\omega / \beta_{2}$. The residues which diminish as $r^{-1 / 2}$ give the normal mode solutions, whereas the branch line integrals diminish as $r^{-2}$, and become negligible for large $r$.

Following this procedure and neglecting for the while the contributions of the branch line integrals, we find for large values of $r$ :

$$
\begin{gathered}
\phi_{1}=\frac{2 \pi}{H} \sqrt{\frac{2}{\pi r}} \sum_{n} \frac{\mathrm{I}}{\sqrt{k_{n}}} \frac{\sin \left(\xi_{n} z\right)}{\xi_{n}} e^{i\left(\omega t-k_{n} r-\omega / 4\right)} \\
\frac{k_{n} H\left\{\frac{\rho_{1}}{\rho_{2}} \frac{\omega^{4}}{\beta_{2}{ }^{4}} \frac{\eta_{n}}{\xi_{n}} \sin \xi_{n}(H-d)-\left[4 k_{n}{ }^{2} \eta_{n} \xi_{n}-\left(2 k_{n}{ }^{2}-\frac{\omega^{2}}{\beta_{2}^{2}}\right)^{2}\right] \cos \xi_{n}(H-d)\right\}}{\frac{\partial}{\partial k}\left\{\frac{\rho_{1}}{\rho_{2}} \frac{\omega^{4}}{\beta_{2}^{4}} \frac{\eta_{n}}{\xi_{n}} \sin \left(\xi_{n} H\right)-\left[4 k_{n}{ }^{2} \eta_{n} \xi_{n}-\left(2 k_{n}{ }^{2}-\frac{\omega^{2}}{\beta_{2}^{2}}\right)^{2}\right] \cos \left(\xi_{n} H\right)\right\}} \quad 0 \leqq z \leqq d .
\end{gathered}
$$

Corresponding expressions for $\phi_{1}^{\prime}, \phi_{2}$ and $\psi_{2}$ are readily obtainable. The subscript $n$ indicates that the quantity is to be evaluated at $k=k_{n}$, where $k_{n}$ are the roots of the period equation obtained by setting the denominators of (26)-(29) equal to zero, namely,

$$
\frac{\rho_{1}}{\rho_{2}} \frac{\omega^{4}}{\beta_{2}{ }^{4}} \frac{\eta}{\xi} \tan (\xi H)-\left[4 k^{2} \eta \zeta-\left(2 k^{2}-\frac{\omega^{2}}{\beta_{2}^{2}}\right)^{2}\right]=0
$$

From the exponential factor in (30) it can be seen that the phase velocity $c_{n}$ can be expressed by

$$
c_{n}=\omega / k_{n}
$$

and the normal mode solutions can be written in their final form: 


$$
\begin{aligned}
& \phi_{1}=\phi_{1}{ }^{\prime}=\frac{2 \pi}{H} \sqrt{\frac{2}{\pi r}} \sum_{n} \frac{\mathrm{I}}{\sqrt{k_{n}}} e^{i\left(\omega t-k_{n} r-\pi / 4\right)} \Phi_{1}\left(k_{n}\right) \sin \left(\xi_{n} d\right) \sin \left(\xi_{n} z\right) \\
& 0 \leqq z \leqq H \\
& \phi_{2}=\frac{2 \pi}{H} \sqrt{\frac{2}{\pi r}} \sum_{n} \frac{\mathrm{I}}{\sqrt{k_{n}}} e^{i\left(\omega t-k_{n} r-\pi / 4\right)} \Phi_{2}\left(k_{n}\right) \sin \left(\xi_{n} d\right) e^{-\cdots(z-H)} \\
& z \geqq I I \\
& \psi_{2}=2 \pi \sqrt{\frac{2}{\pi r}} \sum_{n} \frac{\mathrm{I}}{\sqrt{k_{n}}} e^{i\left(\omega t-k_{n} r-\pi / 4\right)} \Psi_{2}\left(k_{n}\right) \sin \left(\xi_{n} d\right) e^{-\zeta(z-H)} \\
& z \geqq H
\end{aligned}
$$

where

$$
\begin{aligned}
& \Phi_{1}=-\frac{\frac{\rho_{1}}{\rho_{2}} \frac{c^{4}}{\beta_{2}^{4}} \frac{\eta_{n}}{\xi_{n}} k_{n} I I}{\sqrt{c^{2} / v_{1}^{2}}-\mathrm{I}\{\} \cos \left(\xi_{n} H\right)} \\
& \Phi_{2}=-\frac{\frac{\rho_{1}}{\rho_{2}} \frac{c^{2}}{\beta_{2}^{2}}\left(2-c^{2} / \beta_{2}^{2}\right) k_{n} H}{\sqrt{c^{2} / \nu_{1}^{2}-\bar{I}}\{\}} \\
& \Psi_{2}=-\frac{\frac{\rho_{1}}{\rho_{2}} \frac{c^{2}}{\beta_{2}^{2}} \frac{\eta_{n}}{\xi_{n}}}{\{\}} \\
& \{\}=\frac{\rho_{1}}{\rho_{2}} \frac{c^{4}}{\beta_{2}{ }^{4}}\left\{\frac{\sin \left(\xi_{n} H\right)}{\sqrt{c^{2} / v_{1}^{2}-\mathbf{I}} \sqrt{\mathbf{I}-c^{2} / \alpha_{2}{ }^{2}}}\left[\mathbf{I}+\frac{\mathrm{I}-c^{2} / \alpha_{2}{ }^{2}}{c^{2} / v_{1}^{2}-\mathrm{I}}\right]\right. \\
& \left.-\left[\frac{k_{n} H \sqrt{\mathrm{I}-c^{2} / \alpha_{2}{ }^{2}}}{\left(c^{2} / v_{1}{ }^{2}-\mathrm{I}\right)} \sec \left(\xi_{n} H\right)\right]\right\}-4\left\{\frac{\sqrt{\mathrm{I}-c^{2} / \beta_{2}{ }^{2}}}{\sqrt{\mathrm{I}-c^{2} / \alpha_{2}{ }^{2}}}\right. \\
& +\frac{\sqrt{1-c^{2} / \alpha_{2}^{2}}}{\sqrt{1-c^{2}} / \beta_{2}^{2}} \\
& \left.+2 \sqrt{\mathrm{I}-\bar{c}^{2} / \alpha_{2}{ }^{2}} \sqrt{\mathrm{I}-\overline{c^{2} / \beta_{2}{ }^{2}}}-2\left(2-c^{2} /{\beta_{2}}^{2}\right)\right\} \cos \left(\xi_{n} H\right)
\end{aligned}
$$

and

$$
\left.\begin{array}{l}
\xi_{n}=k_{n} \sqrt{c^{2} / v_{1}^{2}-I} \\
\eta_{n}=k_{n} \sqrt{\mathrm{I}-c^{2} / \alpha_{2}^{2}} \\
\zeta_{n}=k_{n} \sqrt{\mathrm{I}-c^{2} /{\beta_{2}}^{2}}
\end{array}\right\} .
$$


Equation (29) can be rewritten in the dimensionless form:

$\tan \left(k_{n} H \sqrt{c^{2} / v_{1}^{2}-\mathrm{I}}\right)$

$$
=\frac{\rho_{2}}{\rho_{1}} \frac{\beta_{2}^{4}}{c^{4}} \frac{\sqrt{c^{2} / v_{1}{ }^{2}-\mathrm{I}}}{\sqrt{\mathrm{I}-c^{2} / \alpha_{2}{ }^{2}}}\left[4 \sqrt{\mathrm{I}-c^{2} / \alpha_{2}^{2}} \sqrt{\mathrm{I}-c^{2} /{\beta_{2}{ }^{2}}^{2}}-\left(2-c^{2} /{\beta_{2}}^{2}\right)^{2}\right] .
$$

The discussion of the previous equation given in earlier papers will be repeated here.

This equation defines an implicit relationship between the frequency $f=c k_{n} / 2 \pi$ and the phase velocity $c$, with the elastic constants of the acoustic system as parameters. It is evident that the wave system defined by (33)-(35) will be attenuated for $k_{n}$ complex, the degree of attenuation depending on the magnitude of the imaginary component. The energy loss associated with damped propagation is due to the refraction of waves from the liquid layer into the solid bottom. For the case $\alpha_{2}>\beta_{2}>v_{1}$ which is considered in this paper, undamped propagation will occur for the cases:

$$
\begin{aligned}
I & \alpha_{2}>\beta_{2} \geqq c \geqq v_{1} \\
I I & \alpha_{2}>\beta_{2}>v_{1} \geqq c .
\end{aligned}
$$

Only case $I$ is considered here.

From the form of the period equation (4I), it can be seen that $k_{n} H$ (and hence frequency) is a multiple valued function of phase velocity, each value belonging to a distinct mode of propagation. The summations indicated in (3r)(33) are to be carried out over all the modes $n=\mathrm{I}$ to $n=\infty$. The amplitude factors $\Phi_{1} / \sqrt{k_{n}}$ and $\Phi_{2} / \sqrt{k_{n}}, \Psi_{2} / \sqrt{k_{n}}$ give the strength of excitation in the first and second layer for each mode as a function of the frequency. A plot of $\Phi_{1}$ for the first and second modes appears in Figure 7 as a function of a dimensionless parameter proportional to frequency.

The factor $\sin \left(\xi_{n} d\right)$ shows the influence of the depth of the source on the amplitudes of the different modes. The vertical pressure distribution for each mode is given by the factor $\sin \left(\xi_{n} z\right)$ which is plotted in Figure 8 for the first two modes.

It can be shown (Press \& Ewing, I948b) that the period equation (4r) expresses the condition of constructive interference between plane waves undergoing multiple reflection in the liquid layer at angles of incidence beyond the critical angle of reflection $\theta_{\varepsilon}$, where $\sin \theta_{\varepsilon}=v_{1} / \beta_{2}$. With this simplified point of view each mode represents a different order of interference and the disturbance at a distant point is obtained by the superposition of waves arriving at the point along the oblique ray paths (defined by the angle of incidence $\theta$ ) for which constructive interference occurs. For the case of plane waves the wave number $k$ in the previous discussion can be expressed by $k=(2 \pi / l) \sin \theta$ where $l$ is the wavelength measured along an oblique ray path. 


\section{Generalizaiion for an Arbitrary Pulse}

In a dispersive medium in which an arbitrary initial disturbance occurs, the energy associated with each period is known to propagate with the group velocity given by the formula

$$
U=c+(k H)(d c) / d(k H)
$$

The period equation (4I) was used to calculate numerically the phase velocity as a function of $k H$. The group velocity was subsequently determined from equation (42), using the values of $d c / d(k H)$ obtained by numerical differentiation. The results of these computations for $\beta_{2} \geqq c \geqq v_{1}$ (case I above) are shown in Figures $I, 2$, and 3 , where $c / v_{1}$ and $U / v_{1}$ are plotted as functions of the dimensionless parameter $\gamma=H / l=k H c / 2 \pi v_{1}=H f / v_{1}$ for three cases a) $\rho_{2} / \rho_{1}=2.5, \alpha_{2}=\sqrt{3} \beta_{2}$, $\beta_{2}=3 v_{1}$, b) $\rho_{2} / \rho_{1}=2.5, \alpha_{2}=\sqrt{3} \beta_{2}, \beta_{2}=2 v_{1}$, and c) $\rho_{2} / \rho_{1}=2.0, \alpha_{2}=\sqrt{3} \beta_{2}, \beta_{2}=1.5 v_{1}$, which represent in an approximate way the conditions for basaltic, granitic and sedimentary bottoms for which $\lambda=\mu$ or Poissons constant $\sigma=0.25$. For each case the first two modes are plotted.

A study of these curves reveals that the phase and group velocity of the first mode $(n=\mathbf{I})$ approach the velocity of Rayleigh waves $\left(c_{R}=0.9 \mathrm{I} 94 \beta_{2}\right)$ in the bottom layer as $\gamma \rightarrow \circ$ or as the wavelength becomes very long in comparison to the thickness of the first layer. As $c / v_{1}$ and $U / v_{1} \rightarrow I, \gamma$ approaches a limiting value.

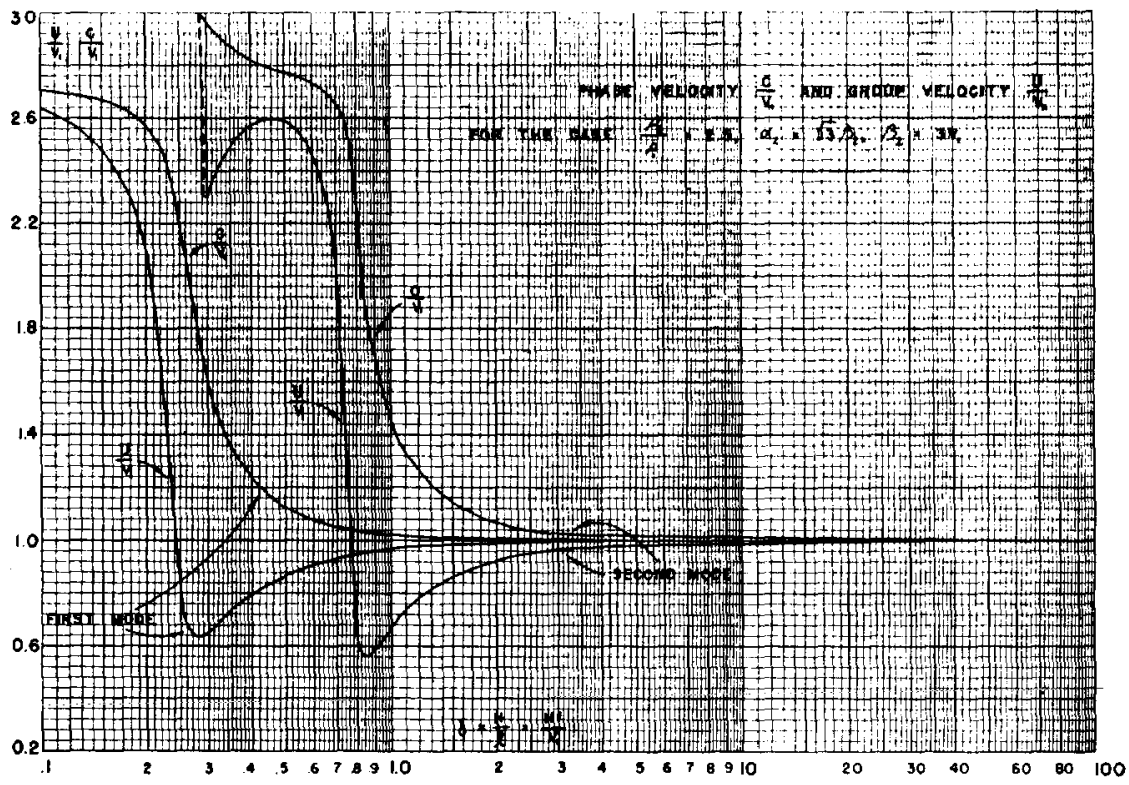

Fig. I. Phase and group velocity curves in the first and second modes for case a. 


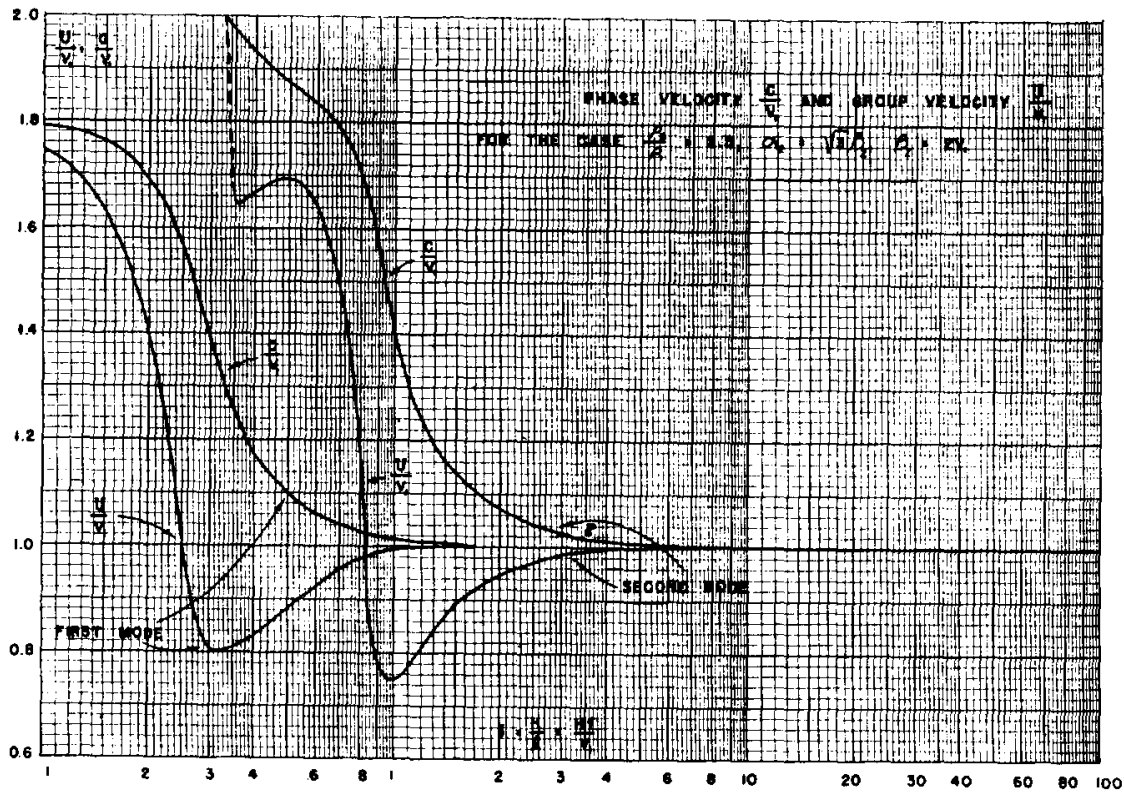

FIG. 2. Phase and group velocity curves in the first and second modes for case b.

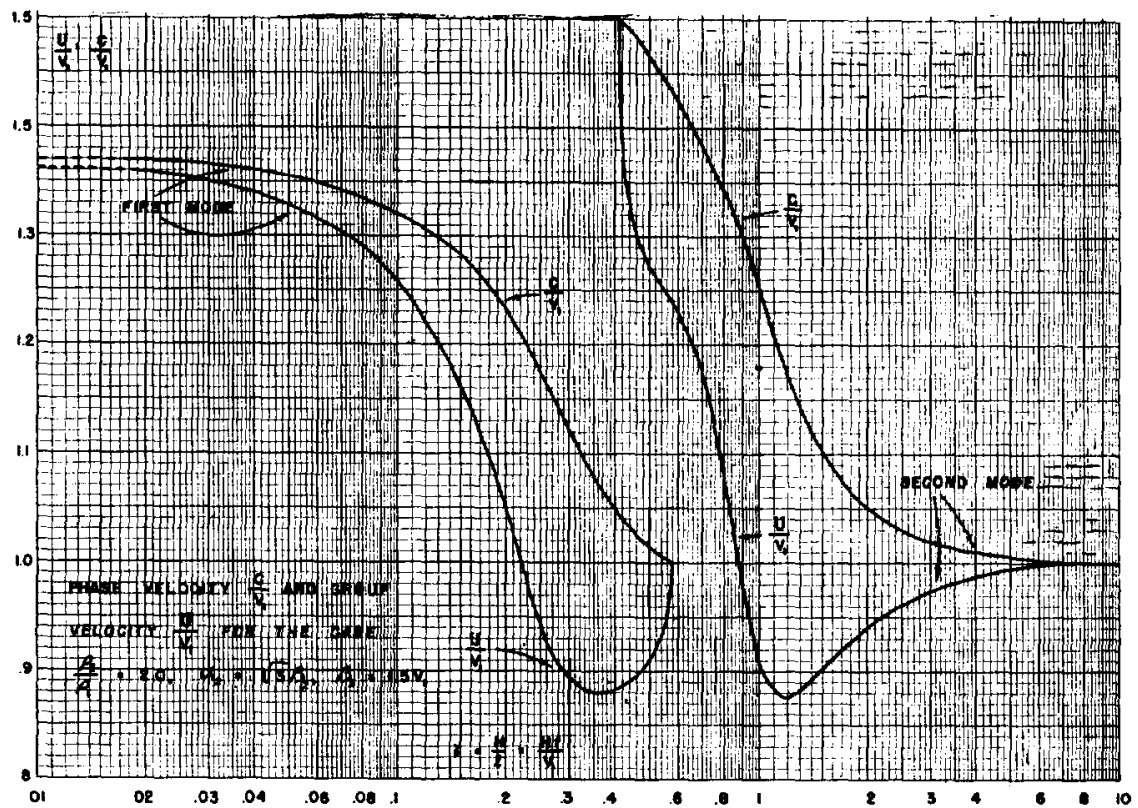

Fig. 3. Phase and group velocity curves in the first and second modes for case c. 
For $c / v_{1}<\mathrm{I}$ (case II above) the propagation reduces to that of a boundary wave (Stoneley wave) along the bottom interface. In the second mode $(n=2)$ the phase and group velocity equals the velocity of shear waves in the bottom $\left(c / v_{1}=U / v_{1}=\beta_{2} / v_{1}\right)$ for a limiting value of $\gamma$ corresponding to a cut-off frequency below which $k$ is complex and damping occurs. For $c$ and $U$ close to $v_{1}$ the frequencies become infinitely large. Higher modes of propagation $(n=3,4, \ldots)$ exist, each having the same cut-off velocity but increasingly large cut-off frequencies. In general the frequencies of the higher modes corresponding to a

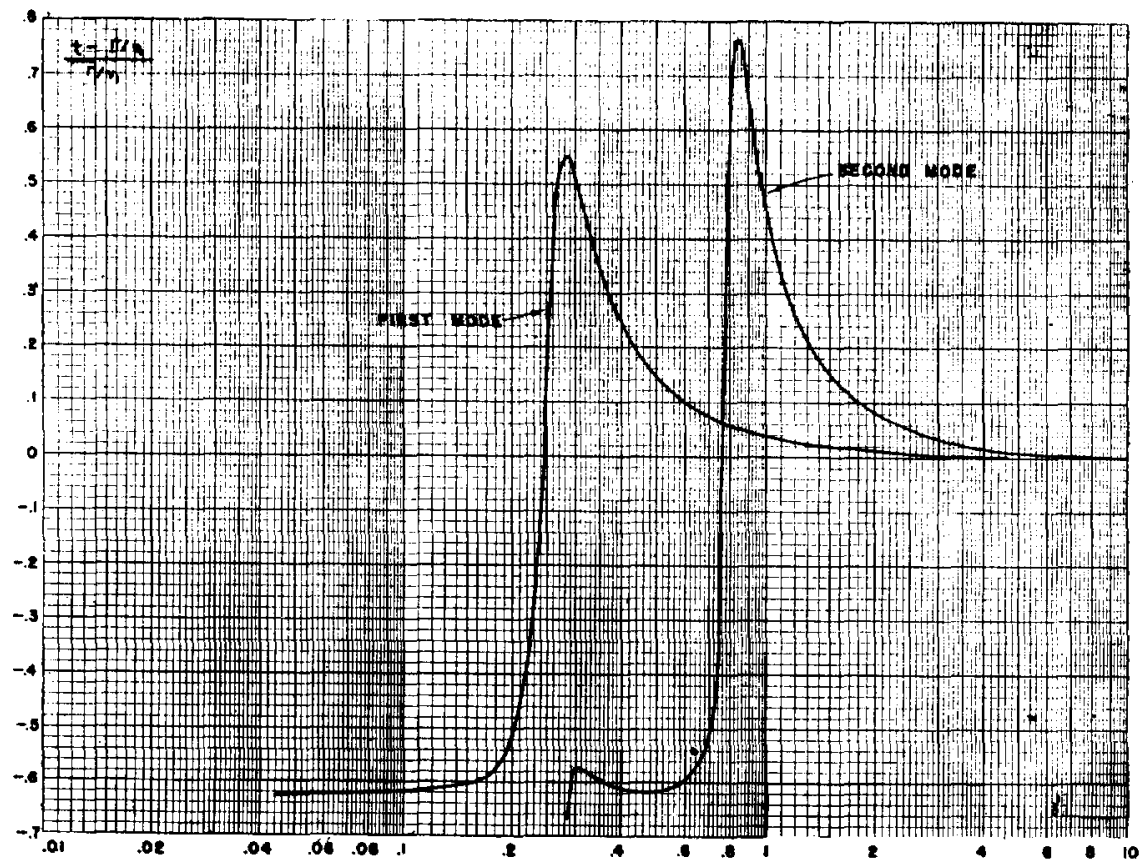

FIG. 4. Dispersion curves relating travel time and frequency for case a.

given phase or group velocity become progressively higher. The group velocity of the first and second modes are further characterized by the existence of stationary values, the importance of which will be discussed in a later paragraph.

Dispersion curves giving frequency as a function of arrival time are given in Figures $4,5,6$ for cases a, b, and c respectively.

Having obtained the solution for the steady state propagation of simple harmonic compressional waves originating in a point source located within the liquid layer, it remains yet to obtain the solution for an arbitrary initial disturbance. If the time variation of the initial disturbance at the source is $f(t)$ represented by its Fourier transform 


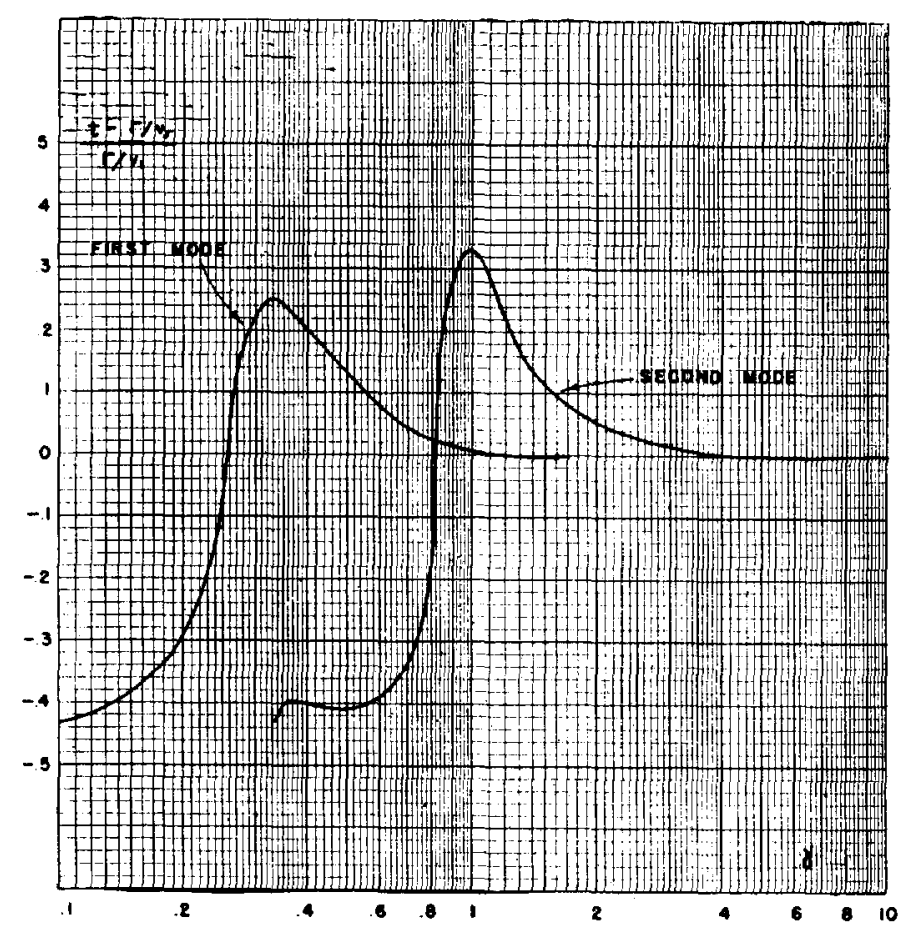

FIG. 5. Dispersion curves relating travel time and frequency for case $b$.

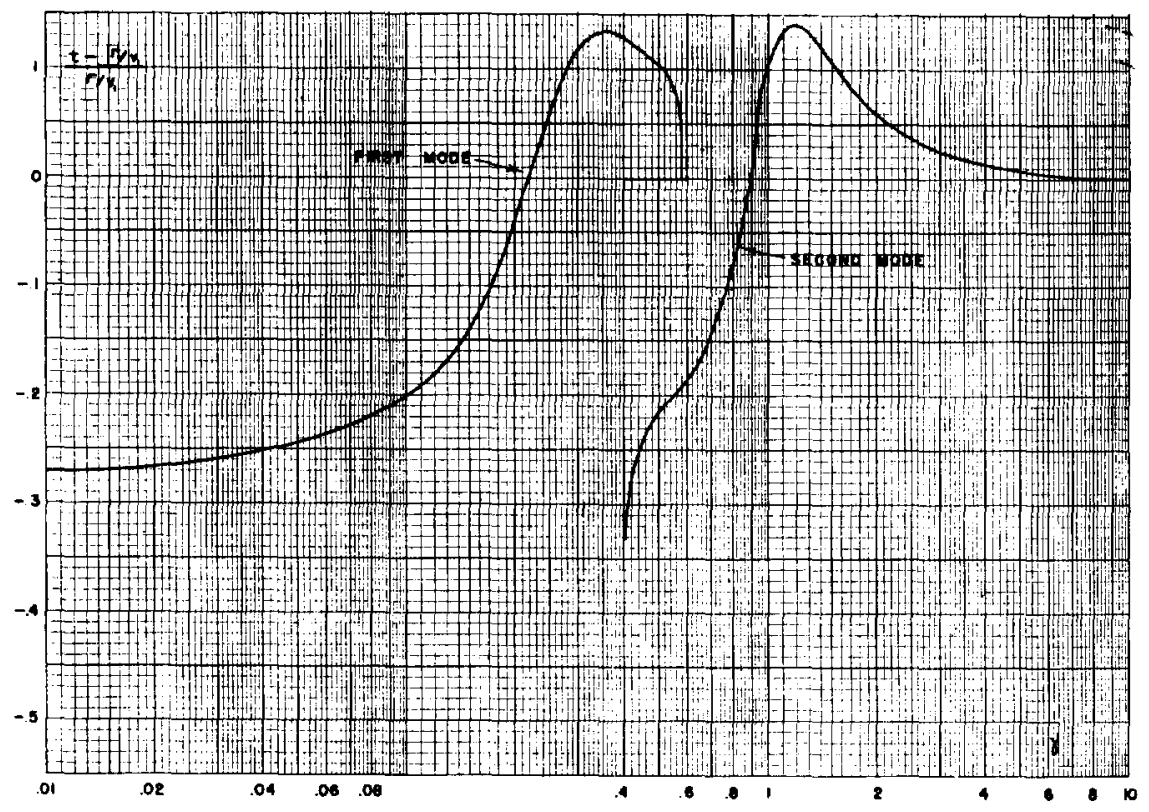

FIG. 6. Dispersion curves relating travel time and frequency for case $c$. 


$$
g(w)=\int_{-\infty}^{\infty} e^{-i \omega} l f(t) d t
$$

then

$$
f(t)=\frac{\mathrm{I}}{2 \pi} \int_{-\infty}^{\infty} e^{i \omega t} g(w) d w
$$

the potential $\phi_{1}$ can be written:

$$
\begin{aligned}
& \phi_{1}=\frac{\mathrm{I}}{H} \sqrt{\frac{2}{\pi r}} \sum_{n} \int_{-\infty}^{\infty} \frac{g(\omega)}{\sqrt{k_{n}}} e^{i\left(\omega t-k_{n} r-\pi / 4\right)} \Phi_{1}\left(k_{n}\right) \sin \left(\xi_{n} d\right) \sin \left(\xi_{n} z\right) d \omega \\
& 0 \leqq z \leqq H
\end{aligned}
$$

The corresponding expressions for $\phi_{2}$ and $\psi_{2}$ can be readily obtained in the same manner. In (45) $k_{n}$ is a function of $\omega$ through (32) and (4I). We follow Pekeris in representing the initial disturbance created in an explosion as follows:

$$
\left.\begin{array}{rlrl}
f(t) & =e^{-\sigma t} & & t>0 \\
& =0 & & t<0
\end{array}\right\}
$$

where

$$
f(t)=\frac{I}{2 \pi} \int_{-\infty}^{\infty} \frac{e^{i \omega t}}{(\sigma+i \omega)} d \omega, \quad g(\omega)=\frac{I}{\sigma+i \omega}
$$

and

$$
\begin{array}{r}
\phi_{1}=\frac{\mathrm{I}}{H} \sqrt{\frac{2}{\pi r}} \sum_{n} \int_{-\infty}^{\infty} \frac{e^{i\left(\omega t-k_{n} r-\pi / 4\right)}}{\sqrt{k_{n}}(\sigma+i \omega)} \Phi_{1}\left(k_{n}\right) \sin \left(\xi_{n} d\right) \sin \left(\xi_{n} z\right) d \omega \\
0 \leqq z \leqq H
\end{array}
$$

$\sigma$ is a parameter that depends primarily on the size of the explosive charge.

To evaluate these integrals we use Kelvin's approximate method of stationary phase. This method assumes that the exponential term is a rapidly oscillating function of $\omega$ over the range of integration, whereas the remainder of the integrand is a slowly varying function of $\omega$. The principal contribution to the integral therefore occurs at the points for wbich $\delta f(\omega) / \delta \omega=0$, where $f(\omega)=\left(\omega t-k_{n} r\right.$ $-\pi / 4)$. An expansion of $f(\omega)$ about these points of stationary phase is made and formulae for the approximate evaluation of the integrals and criteria for the validity of the approximations are obtained. Havelock (I9I4), Jeffreys, (1926) Lamb (1932), and Pekeris (1948) derive and discuss the appropriate formulae. In evaluating (48) we make use of the equations and computations as given by Pekeris and obtain: 


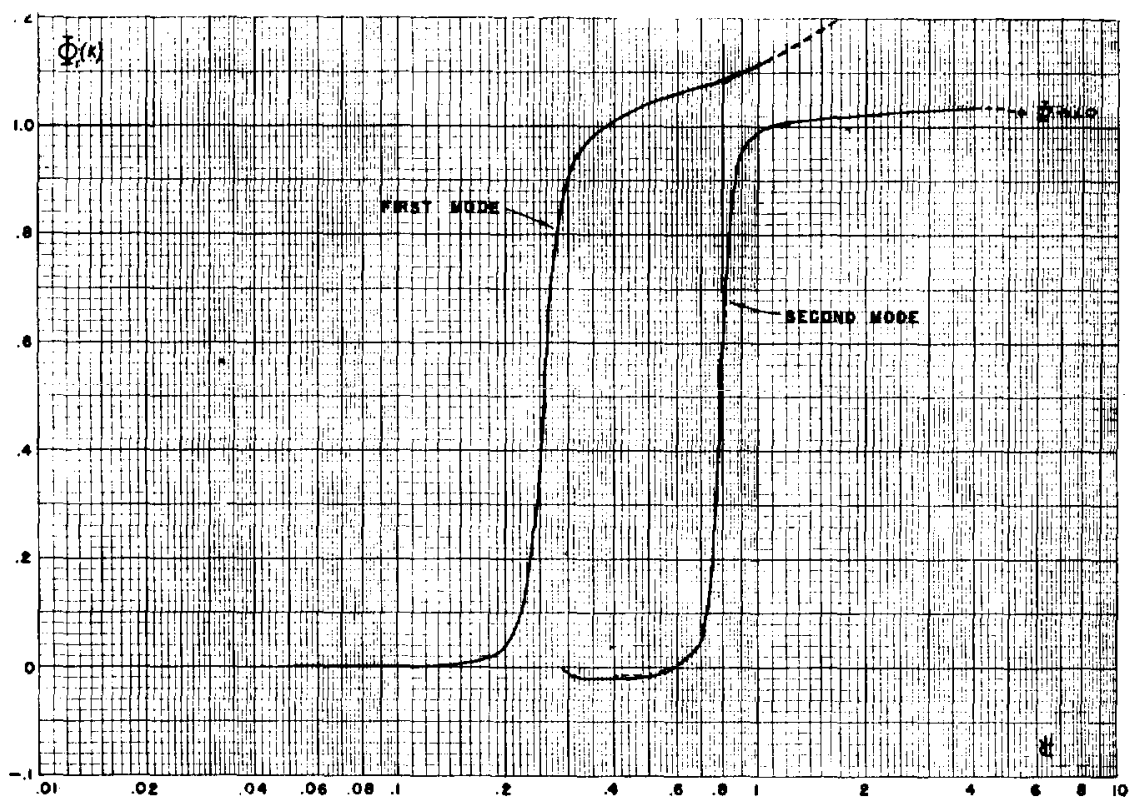

Fig. 7. Excitation function for the first and second modes of case a.
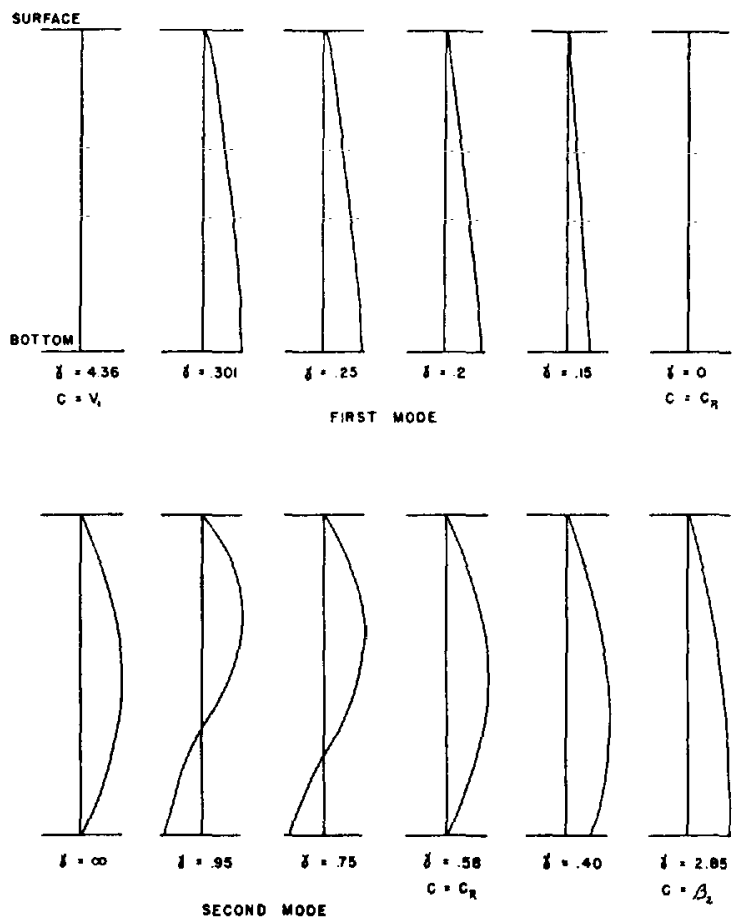

FIG. 8. Vertical pressure distribution in the first and second modes for case a. 


$$
\begin{array}{cc}
\phi_{1}=\frac{2 V_{1}}{H r} \sum_{n} \frac{e^{i\left(\omega_{0} t-k_{n} r-\pi \mid 4 \pm x / 4\right)}}{\left[\frac{\gamma}{c / v_{1}} \frac{\mathbf{I}}{U^{2} / v_{1}^{2}}\left|\frac{d U / V_{1}}{d \gamma}\right|\right]_{n}^{1 / 2}(\sigma+i \omega)} \Phi_{1}\left(k_{n}\right) \sin \left(\xi_{n} d\right) \sin \left(\xi_{n} z\right)(49) \\
0 \leqq z \leqq H
\end{array}
$$

where the upper or lower sign in the exponential term is to be taken according as $d\left(U / v_{1}\right) / d \gamma$ is positive or negative and $k H, U / v_{1}, d\left(U / v_{1}\right) / d \gamma, \omega_{0}$, are evaluated from the phase and group velocity curves of each mode for the values of $r$ and $t$ which satisfy $r / t=U$. Equation (49) is valid provided $r$ is large and $t$ is sufficiently removed from the value $t_{m}=r / U_{m}$ where $U_{m}$ denotes a minimum or maximum value of group velocity. To complete the solution we add to (49) its complex conjugate, since, as Pekeris shows, there are two stationary points $\pm \omega_{0}$ where $\delta f\left(\omega_{0}\right) / \delta \omega=0$ and a solution similar to (49) except for a reversal in sign of the phase of the exponential term would have been obtained had we assumed initially a time factor $e^{-i \omega t}$ instead of $e^{i \omega t}$. Thus we obtain

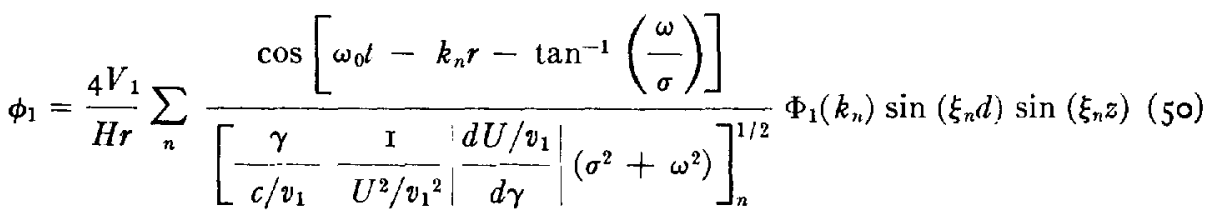
$\circ \leqq z \leqq H$

for

$$
\frac{d U / v_{1}}{d \gamma}>0 \quad \text { and }
$$

$\phi_{1}=\frac{{ }_{4} V_{1}}{H r} \sum_{n} \frac{\cos \left[\omega_{0} t-k_{n} r-\tan ^{-1}\left(\frac{\omega}{\sigma}\right)-\pi / 2\right]}{\left[\frac{\gamma}{c / v_{1}} \frac{\mathbf{I}}{U^{2} / v_{1}^{2}}\left|\frac{d U / v_{1}}{d \gamma}\right|\left(\sigma^{2}+\omega^{z}\right)\right]_{n}^{1 / 2}} \Phi_{1}\left(k_{n}\right) \sin \left(\xi_{n} d\right) \sin \left(\xi_{n} z\right)\left(5^{I}\right)$ $\circ \leqq z \leqq H$

for

$$
\frac{d U / v_{1}}{d \gamma}<0
$$

For $t$ close to $t_{m}=r / U_{m}$, where the group velocity is stationary, a further approximation involving the second derivative as the expansion of $f(\omega)$ is required in order to evaluate (45), giving for large ranges $r$ :

$$
\begin{aligned}
& \phi_{1}=\frac{4 V_{1}}{3^{2 / 3} H r^{\eta / 6}} \sum_{n} \frac{\cos \left[\omega_{0} t-k_{n} r-\tan ^{-1}\left(\frac{\omega}{\sigma}\right)-\pi / 4\right]}{\left[\left(\sigma^{2}+\omega^{2}\right) k / 2 \pi\right]_{n}^{1 / 2}\left[\frac{H^{2}}{2 \pi} \frac{d Z}{d \gamma}\right]_{n}^{1 / 3}} \\
& E(v) \Phi_{1}\left(k_{n}\right) \sin \left(\xi_{n} d\right) \sin \left(\xi_{n} z\right) \\
& 0 \leqq z \leqq H
\end{aligned}
$$


where

$$
\begin{gathered}
E(v)=v^{1 / 3}\left[J_{-1 / 3}(v)+J_{1 / 3}(v)\right] \quad\left\{\begin{array}{l}
t<r / U_{\min } \\
t>r / U_{\max }
\end{array}\right. \\
E(v)=v^{1 / 3}\left[I_{-1 / 3}(v)-I_{1 / 3}(v)\right] \quad\left\{\begin{array}{l}
t>r / U_{\min } \\
t<r / U_{\max }
\end{array}\right. \\
⿱ ⺌=\frac{v_{1}^{2}}{H} \frac{d^{2} k}{d \omega^{2}}=-\frac{v_{1}}{2 \pi U^{2}} \frac{d U}{d \gamma}
\end{gathered}
$$

and

$$
v=\frac{4 \sqrt{\pi}}{3 \sqrt{-\frac{d Z}{d \gamma}}\left(\frac{r}{H}\right)\left(\frac{v_{1}}{U}-\frac{v_{1}}{U_{m}}\right)^{3 / 2}}
$$

Pekeris has given the name Airy phase to waves associated with a maximum value. The factor $E(v)$ represents the envelope of the Airy phase and is plotted

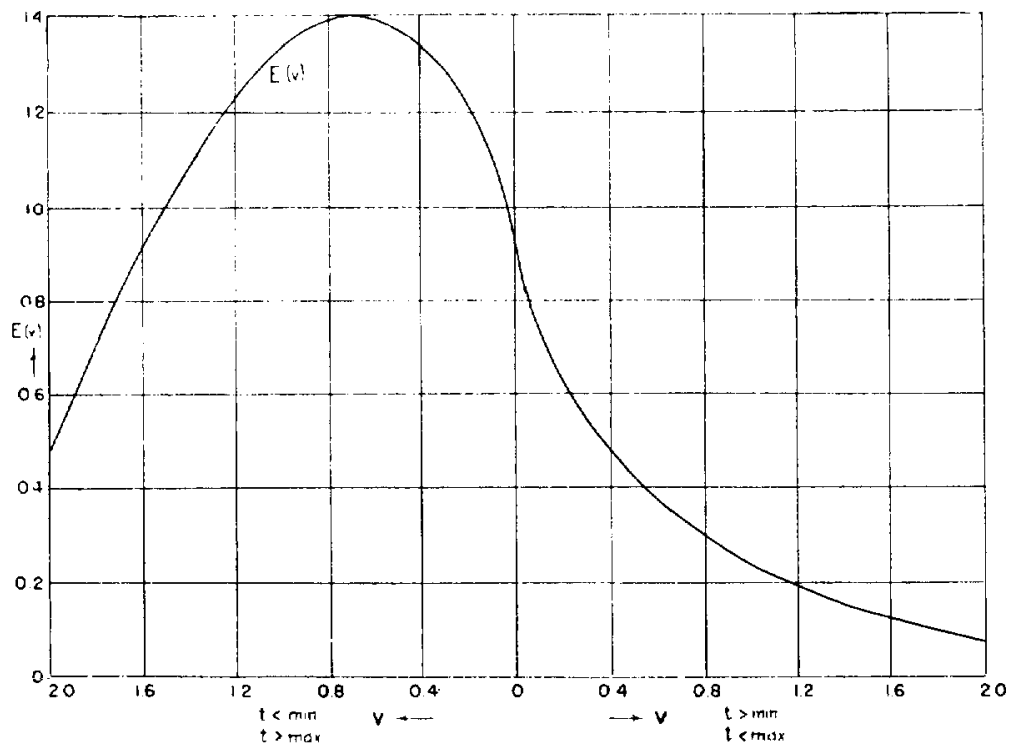

Fig. 9. Envelope of the Airy phase (after Pekeris).

in Figure 9 from the data given by Pekeris. It is to be noted that the Airy phase amplitude falls off only as $r^{-5 / 6}$, in contrast to the $r^{-1}$ decay of waves travelling at other values of group velocity.

The relative amplitudes of the velocity potential $\gamma_{1}$ when both the charge and hydrophone are on bottom can be discussed with the aid of Figure ro where the amplitude factor 


$$
G=\frac{\Phi_{1}\left(k_{n}\right) \sin ^{2}\left(\xi_{n} H\right)}{\left[\frac{\gamma}{c / v_{1}} \frac{\mathbf{I}}{U^{2} / v_{1}^{2}}\left|\frac{d U / v_{1}}{d \gamma}\right|\right]_{n}^{1 / 2}}
$$

obtained by setting $d=z=H$ in equations (50)-(5I) (i.e. assuming charge and receiver on bottom) is plotted as a function of $\gamma$ in the first and second modes for case a in which $\rho_{2} / \rho_{1}=2.5, \alpha_{2}=\sqrt{3} \beta_{2}, \beta_{2}=3 v_{1}$. The relative amplitudes of the Airy phases at the time $t=r / U_{m}$ have been computed for the same conditions and in the same units as $G$ from the factor

$$
A=\frac{r^{1 / 6} E(v) \Phi_{1}\left(k_{n}\right) \sin ^{2}\left(\xi_{n} H\right)}{3^{2 / 3}[k / 2 \pi]_{n}^{1 / 2}\left[H^{2 / 2 \pi}\left(\frac{d Z}{d \gamma}\right)\right]_{1 / 3}^{1 / 3}}
$$

obtained from equation (52). The magnitudes of A for the cases $r=200 \mathrm{H}$ and $r=2000 H$ have been computed and are plotted in Figure ro. Dispersion curves relating the frequency of waves with arrival time are given in Figures $4^{-6}$, so that amplitudes can be obtained as a function of frequency or travel time.

\section{DISCUSSION OF THF: NORMAL MODF SOLUTIONS}

Whether the source is located within the liquid layer or the solid bottom, the dispersion in the normal mode solutions is identical and many of the character-

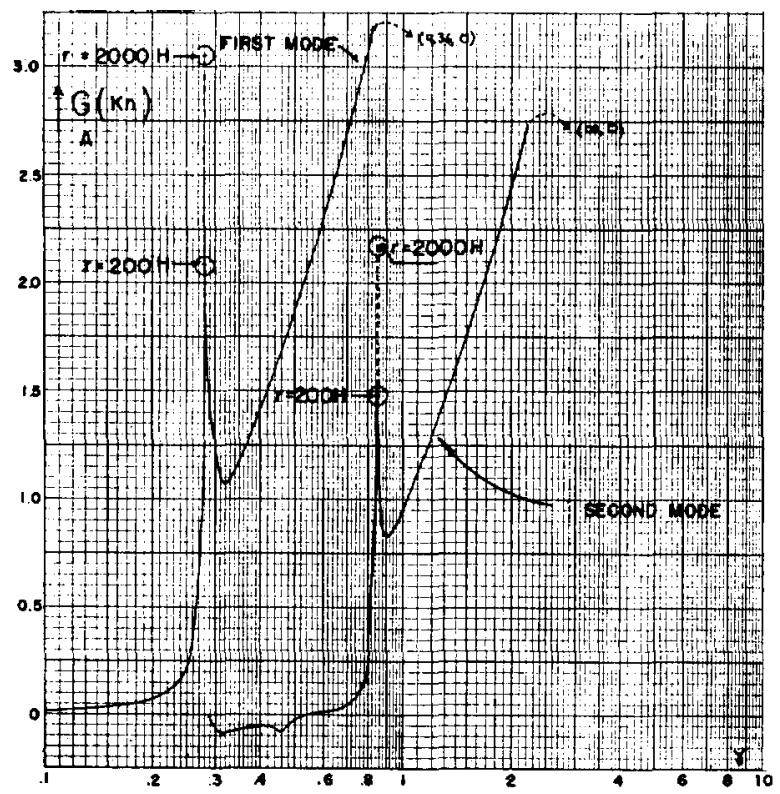

Fig. ro. Amplitude functions in the first and second mode for case a. The circled points give the amplitude of the Airy phases at the distances indicated. 
istics of incoming signals for both problems are similar. The discussion given in an earlier paper which deals with a source within the bottom (Press, et al.) is in the large part applicable and will be repeated here with the slight modification required for a source located within the liquid layer.

In an earlier section the formal steady state solutions (26)-(29) were expressed as the sum of the residues of the integrands and two integrals along branch lines corresponding to the branch points $c=\alpha_{2}$ and $c=\beta_{2}$. The residues lead to the normal mode solutions which predominate at large distances because they diminish only as $r^{-1 / 2}$. Now the normal mode solutions vanish at $c=U=\beta_{2}$ and do not exist for $c>\beta_{2}$. In this region the branch line integrals contribute waves travelling with the speed of compressional and shear waves in the bottom, diminishing rapidly in amplitude with distance as $1 / r^{2}$.

At a time $l>r / \beta_{2}$ after the initial impulse at the source (i.e. immediately after the arrival of shear waves) the normal mode contributions begin, gradually increasing in amplitude to become the predominant waves, providing $r$ is large. The wave motion in the first two modes due to a distant impulsive point source of compressional waves having a moderately broad spectrum and located within the liquid layer can be obtained from Figure ro as a function of frequency, and from Figures 4 and Io as a function of arrival time. In the first mode the group velocity approaches the velocity of Rayleigh waves $\left(C_{R}=.9\right.$ r $\left.94 \beta_{2}\right)$ as $\gamma \rightarrow 0$. The first arrivals consist of low frequency Rayleigh waves whose amplitudes increase gradually from zero as the frequency increases from zero rapidly at first and then gradually. At the time $t=r / v_{1}$ a high frequency wave arrives travelling with the speed of sound in water. The group velocity and dispersion curves show an upper frequency limit for these waves. For frequencies above this limit the solutions reduce to boundary waves propagated along the interface in a manner analogous to Stoneley waves. According to Figures 4 and ro the amplitudes of these waves are zero at $t=r / v_{1}(\gamma=4.36)$ but increase to large amplitudes shortly thereafter.

For $t>r / v_{1}$ the high frequency and low frequency branches of the group velocity curve contribute waves which arrive simultaneously and approach each other in frequency until they merge to form an Airy phase at a time corresponding to propagation at the minimum value of group velocity. The Airy phase amplitude of the first mode has been calculated from equation $\left(5^{6}\right)$ and is plotted in Figure Io for the two cases $r=200 H$ and $r=2000 H$. It is seen that Airy phase waves have large amplitudes relative to waves travelling at other values of group velocity, the amplitude of the Airy phase increasing with range as $r^{1 / 6}$ relative to waves traveling at other values of group velocity.

The second mode begins with waves arriving with a cut-off frequency at the time $t=r / \beta_{2}$. The amplitudes are zero at the onset and thereafter increase as the frequency increases. At the time $t=r / v_{1}$ high frequency waves $(\gamma \rightarrow \infty)$ arrive travelling with the speed of sound in water. The amplitude of these waves is zero at the onset but increases rapidly thereafter. For $l>r / v_{1}$ the two arrivals corresponding to the low and high frequency branches of the group velocity curve of 
this mode approach each other and merge at a minimum value of group velocity, producing the large amplitude waves of an Airy phase. The second mode Airy phase occurs at higher frequencies and has somewhat smaller amplitudes than that of the first mode.

It is to be noted that a maximum value of group velocity is present in the second mode. Ordinarily one might expect large amplitude waves to occur here, but the excitation function $\Phi_{1}(K)$ (Fig. 7) almost vanishes for the value of $k H$ corresponding to this stationary value of group velocity and the resultant amplitudes show only a minor increase.

From equations (2), (3), (50), (5I) it can be seen that the factor $\sin \left(\xi_{n} z\right)$ gives the vertical variation of pressure and horizontal displacement and $\cos \left(\xi_{n} z\right)$ gives the vertical variation of vertical displacement in the liquid layer. Similarly the factors $e^{-S_{n}(z-H)} e^{-\eta_{n}(z-H)}$ give the vertical variation of stress and displacement in the bottom layer. Nodes of pressure correspond to antinodes of vertical displacement and vice versa. In Figure 8 the vertical variation in amplitude of pressure in the liquid layer is presented as a function of $\gamma$ or the first two modes.

The wave motion at a point is obtained by the superposition of the contributions of all modes. Our discussion thus far has been limited to the first two modes. The frequencies of the higher modes corresponding to a given value of group velocity become progressively larger. In addition, the contributions of the high frequency higher modes will be cut down because of their greater susceptibility to scattering by an irregular bottom. It is only with a low pass receiver system however that a record uncomplicated by the contributions of the higher modes can be obtained. For a more detailed discussion of this point, the reader is referred to the paper of Pekeris ( 1948 ).

\section{APPLICATION TO REFRACTION SHOOTING IN WATER COVERED AREAS}

From the theory just presented a number of important conclusions can be drawn concerning the propagation of explosion sound over large ranges in water covered areas.

I) For solid bottoms, the amplitudes of waves travelling with the speed of compressional waves in the bottom will be relatively small. It is only after the arrival of the first shear waves that large amplitude waves appear. The shear waves begin with a limiting or cut-off frequency which is characteristic of the depth of water and the elastic constants of the bottom. For bottoms which can be treated as liquid, waves having appreciable amplitudes appear shortly after the arrival of the bottom compressional or ground waves. These ground waves begin with a cut-off frequency in a manner analogous to the shear waves of the solid bottom theory.

2) For a solid bottom a train of very low frequency waves $(\gamma \rightarrow 0)$ travelling with the speed of Rayleigh waves in the bottom arrives. These waves increase in frequency and amplitude with increasing time. There is no analogous arrival in the liquid bottom theory. 
3) For both the liquid and solid bottom theory a high frequency wave travelling with the speed of sound in water arrives riding on a low frequency "rider" wave. The frequency of the water waves shows a marked decrease with time. For the liquid bottom the amplitude of the water wave gradually increases with time. For the solid bottom the water waves rise to large amplitudes immediately after they arrive and then fall off somewhat.

4) For both the liquid and solid bottom theory the water waves and rider waves merge to form a train of waves of large amplitude which is known as an Airy phase. The frequency of the Airy phase is determined by the depth of water and the elastic structure of the bottom. The velocity of the Airy phase depends only on the elastic constants in the bottom.

5) The response of a hydrophone sensitive to pressure changes and a geophone sensitive to the vertical velocity of a water particle will vary with depth because of the vertical standing wave pattern shown in Figure 8. For any given mode and frequency the ideal location of a hydrophone is at a pressure antinode and the ideal location of a geophone is at an antinode of vertical displacement. Antinodes and nodes for pressure correspond respectively to nodes and antinodes for vertical displacement (or velocity). With the use of curves such as those of Figure 8 the vertical location of a receiver for peak response at a given frequency can readily be obtained.

In all water covered areas where refraction shooting is undertaken, layering in the bottom occurs and our assumption of an unstratified bottom is indeed an over-simplification. If the thickness of the first bottom layer is of the order of several times the water depth, the above theory should be applicable to a fair degree of approximation.

\section{ACKNOWLFDGMENT}

The research reported in this document has been made possible through support and sponsorship extended by the Geophysical Research Directorate of the Cambridge Field Station, AMC, U.S. Air Force, under Contract W-28-099 ac-396. It is published for technical information only and does not represent recommendations or conclusions of the sponsoring agency.

\section{BIBLIOGRAPHY}

Havelock, T. H., "The Propagation of Disturbances in Dispersive Media," Cumbridge Trucls in Mathemulics and Mathematical Physics No. 17, Cambridge University Press, 1914.

Jeffreys, Harold, "On Surface Waves of Earthquakes," Mon. Not. Roy. Astron. Soc., Geophysicul Suppl. I (1926) $282-203$.

Lamb, Horace, "On the Propagation of Tremors over the Surface of an Elastic Solid," Phil. Trans., Roy. Soc. Lon., Series A, 203 (IgO4) I-42.

--_-_ Hydrodynamics, Cambridge University Press, 6th Ed., (1932) pp. 395-398.

Lee, A. W., "Further Investigations of the Effect of Geological Structure upon Microseismic Disturbance," Mon. Not. Roy. Astron. Soc., Geophys. Suppl., 3 (1934) 238-252.

Nakano, H., "On Rayleigh Waves," Jap. Journ. Astron. and Geophys., II, 5 (1925) 233-326. 
Pekeris, C. L., "Theory of Propagation of Explosive Sounds in Shallow Water, in Propagation of Sound in the Ocean," Mem. No. 27, Geol. Soc. Amer., 1948.

Press, Frank and Maurice Ewing, "Low Speed Layer in Water Covered Areas," Geophysics, 13, No. 3, (1948a) 404-420.

- "A Theory of Microseisms with Geological Applications," Trans. Amer. Geoph. Union, 29, No. 2 (1948b) $163-174$.

Press, Frank, Maurice Ewing and Ivan Tolstoy, "The Airy Phase of Shallow Focus Submarine Earthquakes," Columbia University Technical Report on Seismology No. 3 (Also in press Bull. Seis. Soc. Amer:).

Sezawa, Katsutada, "On the Transmission of Seismic Waves on the Bottom Surface of an Ocean," Bull. Eurth Inst. Tokyo, 9 (1934) I I 5-143.

- - "Love-Waves Generated from a Source of a Certain Depth," Bull. Earth Inst. Tokyo, 13, Pt. I (I935) I-I 7 .

Scholte, J. G., "Over Het Verband Tussen Zeegolven en Microseismen, I and II," Verslag Ned. A kad. Wet., 52 (1943) 669-683.

Stoneley, Robert, "The Effect of the Ocean on Rayleigh Waves," Mon. Not. Roy. Astron. Soc., Geophys. Suppl., I (1926) 349-356.

Worzel, J. L. and Maurice Ewing, "Explosive Sounds in Shallow Water, in Propagation of Sound in the Ocean," Mem. No. 27, Geol. Soc. Amer., 1948. 